\title{
Manejo infectológico de la aplasia medular severa en pacientes pediátricos
}

\author{
Recibido: 22/11/17 Aceptado: 22/12/17
}

Andrea Mónaco ${ }^{1}$

\section{RESUMEN}

La aplasia medular es una enfermedad poco frecuente en pediatría, siendo el tratamiento de elección en las formas severas el trasplante de células progenitoras hematopoyéticas (TCPH). Gracias a los avances en TCPH, los nuevos tratamientos inmunosupresores y al adecuado tratamiento de sostén, se ha logrado en las últimas décadas una franca disminución de la mortalidad asociada a esta patología. Es por ello que uno de los principales desafíos consiste en prevenir la aparición de infecciones asociadas a la neutropenia severa y prolongada que padecen estos pacientes, siendo actualmente las infecciones bacterianas y fúngicas una de las principales causas de morbimortalidad. Por otra parte, la mayoría de las guías de manejo y tratamiento de sostén se basan en recomendaciones de expertos, siendo la evidencia escasa, más aún en pediatría. Gran parte de las recomendaciones de tratamiento empírico se basan en guías de neutropenia febril de pacientes hemato-oncológicos. A su vez, existe gran variabilidad, de acuerdo al centro de atención, en cuanto al uso de antimicrobianos para profilaxis primaria, debiéndose tener en cuenta la mayor propensión a presentar infecciones invasivas por hongos filamentosos $y$, en el caso de pacientes con linfopenia marcada, de enfermedad por $P$ jirovecii a la hora de valorar la indicación de profilaxis de estos pacientes.

Se detallarán a continuación las principales recomendaciones sobre manejo de prevención de infecciones y tratamiento precoz de pacientes pediátricos con aplasia medular severa.
${ }^{1}$ Servicio de Epidemiología e Infectología. Hospital de Pediatría SAMIC "Prof. Dr. Juan Pedro Garrahan".

Dirección de correspondencia: Dra. Andrea Mónaco. Servicio de Epidemiología e Infectología. Hospital de Pediatría SAMIC "Prof. Dr. Juan Pedro Garrahan". Combate de los Pozos 1881, CABA, Argentina. monaco_rodriguez@yahoo.com.ar

La autora manifiesta la inexistencia de algún tipo de conflicto de intereses.

Palabras clave: anemia aplásica, aplasia medular en pediatría, profilaxis antimicrobiana, prevención de infecciones, tratamiento empírico. 


\section{Introducción}

La anemia aplásica es una enfermedad poco frecuente de aparición bimodal que se presenta tanto en etapas tempranas como tardías de la vida. Su incidencia estimada es de dos a cuatro casos por 1.000 .000 de niños menores de 15 años (1). Aunque es relativamente rara en la infancia, su impacto médico sigue siendo alto, con consecuencias potencialmente mortales que implican terapias médicas complejas y posibles complicaciones a largo plazo.

Se considera aplasia medular severa cuando la celularidad en la médula ósea (celularidad central) es menor al $25 \%$, con al menos dos de los siguientes criterios en sangre periférica: recuento de neutrófilos menor a $0,5 \times 10^{9} / \mathrm{L}$, recuento de plaquetas menor a $20 \times 10^{9} / \mathrm{L}$ o reticulocitos menores a 20 $x 10^{\%} / \mathrm{L}$. Cuando el recuento de neutrófilos es $<0,2 \times 10^{9} / \mathrm{L}$ se la define como muy severa (2).

Los síndromes de fallo medular pueden ser congénitos (15-20\%) o adquiridos. Las aplasias adquiridas pueden ser secundarias a exposición ambiental (tóxicos, radiaciones, infecciones virales, enfermedades neoplásicas, otras causas) o por enfermedad primaria de la médula ósea (idiopática), siendo esta última la de mayor frecuencia de aparición. Tanto las formas hereditarias como las adquiridas pueden manifestarse en la niñez como en etapas posteriores de la vida, siendo indispensable su correcto diagnóstico, ya que presentan un diferente abordaje terapéutico (2).

En los pacientes pediátricos uno de los principales diagnósticos diferenciales de la anemia aplásica adquirida es la anemia de Fanconi, un trastorno autosómico recesivo, que en el $60-70 \%$ de los casos se asocia a otras anomalías congénitas $(3,4)$. Otros síndromes congénitos a descartar son la disqueratosis congénita y la anemia de Blackfan Diamond.

El tratamiento de elección es el trasplante de células progenitoras hemopoyéticas (TCPH) de donante familiar histoidéntico, con el que se ha obtenido una mejor respuesta en niños que en adultos $(5,6)$. En un trabajo donde se incluyeron 1300 pacientes con aplasia medular, trasplantados entre 1991 y 2004, se observó que la supervivencia a los cinco años para los menores de 20 años fue del $82 \%$, en tanto que para los que tenían entre 20 y 40 años fue del $72 \%$ y para los mayores a 40 años fue solo del $53 \%$ (7).

Se estima que la supervivencia libre de enfermedad a los diez años en niños post trasplante es del 64 al 97\% (8).
Otra opción terapéutica es el tratamiento médico inmunosupresor, con tasas estimadas de supervivencia a los diez años de $80 \%$ en algunas series de casos (9). Se encuentra principalmente indicado en pacientes que no cuenten con un donante histoidéntico relacionado.

Las tasas de respuesta publicadas son de $50-70 \%$. La respuesta es evidente dentro de los 3-4 meses de iniciado el tratamiento. Un número importante de pacientes mejora la calidad de la respuesta a los seis meses.

Las tasas de mortalidad en niños con aplasia han disminuido en forma contundente en las últimas décadas. En los años 70 la mortalidad al año, solo con tratamiento de sostén, era mayor al $80 \%$. Con los actuales tratamientos las tasas de supervivencia publicadas son del 70 a $100 \%$ a los dos y diez años (3).

En los casos refractarios se requiere un manejo adecuado, siendo las infecciones bacterianas y fúngicas las principales causas de morbimortalidad en pacientes con aplasia medular severa $(10,11)$. Las complicaciones infecciosas se encuentran fundamentalmente asociadas a la neutropenia persistente y al descenso de los monocitos, incrementándose el riesgo de infección y mortalidad en niños con neutropenia severa (<200 NT) (12). Estos pacientes tienen un riesgo alto de presentar infecciones fúngicas invasivas, particularmente las ocasionadas por hongos filamentosos como Aspergillus $\operatorname{spp}(13,14)$.

Por estas razones resulta imprescindible que estos pacientes tengan un adecuado manejo clínico-infectológico, mediante la instauración oportuna del tratamiento de las infecciones asociadas a la neutropenia prolongada, junto con la adecuada valoración de la profilaxis antimicrobiana y la prevención de infecciones a través de un ambiente protegido. Por otra parte se requiere una mayor evidencia en pediatría para poder establecer un mayor grado de recomendación en las guías de manejo de pacientes con aplasia medular.

\section{Prevención de infecciones Ambiente seguro}

En el caso de pacientes internados se recomienda, al igual que en los pacientes oncológicos, utilizar las precauciones estándares, respetando el lavado de manos antiséptico antes y después del contacto con el paciente y su entorno. El uso de equipos de protección (guantes, barbijo y antiparras) se recomienda en caso de contacto con fluidos 
corporales. Se implementarán medidas de precaución respiratoria o de contacto cuando el tipo de infección así lo requiera. Se recomienda, a su vez, la permanencia de los pacientes en habitaciones con control del aire y, en caso de ser trasladados fuera de la habitación, deberán utilizar barbijos de alta eficiencia.

Por otra parte debe evitarse el contacto con áreas de construcción por el riesgo de infecciones fúngicas invasivas por hongos filamentosos (10).

El equipo de salud deberá estar adecuadamente vacunado contra enfermedades inmunoprevenibles y deberá evitar el contacto con los pacientes en caso de presentar enfermedades transmitidas por aire o por contacto directo, hasta que su cuadro infeccioso se encuentre resuelto.

En relación a la alimentación, es recomendable el lavado de manos previo a la preparación de los alimentos. En los pacientes con aplasia severa se recomienda el consumo de alimentos pasteurizados, evitar la ingesta de carne, huevos y pescados crudos, vegetales no lavados y frutas secas. La comida deberá refrigerarse evitando el contacto de los alimentos crudos con los cocidos (10).

Por otra parte, la higiene bucal debe realizarse con un cepillo suave para evitar lesiones de la mucosa y sangrados, pudiendo agregarse antisepsia con enjuagues bucales.

\section{Profilaxis antimicrobiana}

Profilaxis antibiótica

Se encuentra dirigida a prevenir las infecciones por bacilos Gram negativos (BGN). Dentro de los antibióticos estudiados en pacientes adultos hemato-oncológicos con neutropenia severa, las quinolonas se asociaron a una reducción de la mortalidad (15). En similar población pediátrica un estudio reportó, tras la profilaxis con ciprofloxacina, una disminución de los episodios de neutropenia febril (16). En otros estudios la profilaxis con ciprofloxacina se asoció a un menor número de bacteriemias, requiriéndose un mayor número de pacientes y seguimiento a largo plazo para constatar su efectividad y la aparición de resistencia en la flora bacteriana intestinal $(17,18)$.

En los pacientes con aplasia medular no hay uniformidad en relación al uso de fluoroquinolonas para profilaxis $(3,19)$. En un relevamiento realizado en EE.UU. sobre 18 centros pediátricos el $22 \%$ no utilizaba antibióticos profilácticos. En el caso de los que si la utilizaban, esta era discontinuada cuando se alcanzaban recuentos de neutrófilos >500 (20).
No hay aún suficiente evidencia para recomendar su uso en aplasia medular.

\section{Antifúngicos}

Debido al riesgo acrecentado de infecciones fúngicas invasivas por hongos filamentosos, algunos centros utilizan antifúngicos en forma profiláctica (19). Sin embargo, no hay recomendaciones uniformes ni beneficios clínicos demostrados en los pacientes con aplasia medular (10). Deberá considerarse la profilaxis primaria en pacientes con neutropenia severa (NT menor a 200) y prolongada (mayor a 7 días). Se recomienda utilizar drogas activas contra hongos filamentosos, como posaconazol (mayores de 13 años), voriconazol e itraconazol, debiendo realizarse el dosaje de las mismas entre del quinto al séptimo día de iniciada la profilaxis (20). El fluconazol no provee cobertura contra Aspergillus spp, por lo cual a pesar de que algunos centros lo utilizan, no estaría recomendado (4).

\section{Profilaxis contra P. jirovecii}

No está indicada en forma rutinaria. Se recomienda su uso posterior al tratamiento inmunosupresor y en aquellos pacientes con linfocitos totales menores a 1000 o recuento de CD4 menor a 400/mm3, extendiéndose la misma hasta la recuperación de linfocitos y luego de tres meses de finalizado el tratamiento inmunosupresor. En algunos centros $(3,6,19$, 21) se utiliza pentamidina inhalatoria o dapsona, debido a que presenta menor toxicidad medular en comparación con trimetoprima sulfametoxasol trisemanal.

\section{Citomegalovirus (CMV) y Epstein-Barr (EBV)}

No estaría indicado el monitoreo rutinario de la carga viral. El riesgo de reactivación estaría fundamentalmente asociado a regímenes más inmunosupresores (alemtuzumab, timoglobulina de conejo), en cuyo caso sí se sugeriría solicitar PCR cuantitativa para CMV y VEB en pacientes con fiebre sin foco y sin documentación microbiológica.

Se sugiere el uso de productos CMV (-) o desleucotizados por filtración en pacientes con serología desconocida o negativa para CMV, siendo de relevancia determinar su status serológico.

Herpes simplex virus tipo 1 (HSV-1) y varicela zoster virus (VZV)

No se recomienda la profilaxis para HSV-1 y 2.

En caso de contacto con varicela indicar profilaxis post exposición dentro de las 96 hs con gammaglobulina 
específica (VZIG) IM, 125 Ul cada 10 kilogramos de peso (dosis límite: 625 unidades), gammaglobulina estándar (IGIV) EV $400 \mathrm{mg} / \mathrm{k}$ o aciclovir (VO) desde el séptimo día de la exposición, durante siete días.

En caso de infección por HSV-1/2 o VZV se deberá indicar tratamiento $\mathrm{EV}$ con aciclovir.

\section{Influenza}

En pacientes con aplasia severa y post tratamiento con terapia con timoglobulina se recomienda la profilaxis post exposición con oseltamivir, dentro de las 48 hs del contacto y por diez días (10). Indicar tratamiento en caso de infección probada.

\section{Tratamiento empírico de infecciones fúngicas y bacterianas}

Debido a la falta de evidencia para realizar recomendaciones sobre el manejo de los episodios febriles en niños con aplasia medular, el mismo se deberá realizar siguiendo los lineamientos de las guías pediátricas de neutropenia febril en pacientes oncohematológicos. El tratamiento empírico inicial deberá ser de amplio espectro y basado en la epidemiología de cada institución. El esquema antibiótico se ajustará de acuerdo a los hallazgos microbiológicos y a la evolución clínica del paciente, no debiendo discontinuarse la cobertura para BGN, mientras persista el paciente febril, debido al riesgo de superinfección.

En el caso de pacientes con neutropenia febril persistente se deberá realizar tratamiento empírico precoz con drogas activas contra Aspergillus spp (formulaciones lipídicas de anfotericina, voriconazol, caspofungina), junto con el dosaje de galactomananos y TC de tórax y senos paranasales, evaluando, según los resultados, la realización de procedimientos invasivos

De igual modo, en caso de infección probada o posible por Aspergillus spp se deberá iniciar precozmente el tratamiento, continuando luego con profilaxis secundaria.

\section{Referencias}

1. Guinan EC. Acquired aplastic anemia in childhood. Hematol Oncol Clin N Am 23 2009; 23(2): 171-191.

2. Sociedad Argentina de Hematología. Síndromes de fallo medular. http://www.sah.org.ar/docs/363-394.8.SAH_ GUIA2012_FalloMedular.pdf.

3. Marsh JC, Ball SE, Cavenagh J, Darbyshire P, Dokal I, Gordon-Smith EC, et al. British Committee for Standards in Haematology. Guidelines for the diagnosis and management of aplastic anaemia. Br J Haematol 2009; 147(1):43-70.

4. Bacigalupo A, Passweg J. Diagnosis and treatment of acquired aplastic anemia. Hematol Oncol Clin North Am. 2009; 23(2):159-70.

5. Locasciulli A, Oneto R, Bacigalupo A, Socié G, Korthof E, Bekassy A, et al. Severe Aplastic Anemia Working Party of the European Blood and Marrow Transplant Group. Outcome of patients with acquired aplastic anemia given first line bone marrow transplantation or immunosuppressive treatment in the last decade: a report from the European Group for Blood and Marrow Transplantation (EBMT). Haematologica 2007; 92(1):118.

6. Scheinberg P, Young NS. How I treat acquired aplastic anemia. Blood 2012; 120(6):1185-1196.

7. Gupta V, Eapen M, Brazauskas R, Carreras J, Aljurf M, Gale RP, et al. Impact of age on outcomes after bone marrow transplantation for acquired aplastic anemia using HLA-matched sibling donors. Haematologica 2010; 95(12):2119-25.

8. Kojima S1, Horibe K, Inaba J, Yoshimi A, Takahashi Y, Kudo $\mathrm{K}$, et al. Long-term outcome of acquired aplastic anaemia in children: comparison between immunosuppressive therapy and bone marrow transplantation. British Journal of Haematology 2000; 111(1): 321-328.

9. Scheinberg $\mathrm{P}, \mathrm{Wu} \mathrm{C} \mathrm{O}$, Nunez $\mathrm{O}$, Young $\mathrm{N}$. Long term outcome of pediatric patients with severe aplastic anemia treated with anti-thymocyte globulin and cyclosporine. J Pediatr 2008; 153(6): 814-819.

10. Höchsmann B, Moicean A, Risitano A, Ljungman P, Schrezenmeier $H$. Supportive care in severe and very severe aplastic anemia. Bone Marrow Transplantation 2013; 48(2): 168-173.

11. Quarello P, Saracco P, Giacchino M, Caselli D, Caviglia I, Longoni $D$, etal. Epidemiology of infections in children with acquired aplastic anaemia: a retrospective multicenter study in Italy. Eur J Haematol. 2012; 88(6):526-34.

12. Valdez J, Scheinberg $P$, Nunez $O$, Wu C, Young N, Walsh T. Decreased infection-related mortality and improved 
survival in severe aplastic anemia in the past two decades. Clin Infect Dis 2011; 52(6):726-35.

13. Kako $S$, Nakasone $H$, Endo $H$, Sakamoto $K$, Ashizawa $M$, Sato $M$, et al. Clinical course of patients with aplastic anemia or myelodysplastic syndrome associated with persistent neutropenia. Hematol Oncol 2012; 30(2): 8288.

14. Torres HA1, Bodey GP, Rolston KV, Kantarjian HM, Raad II, Kontoyiannis DP. Infections in patients with aplastic anemia: experience at a tertiary care cancer center. Cancer 2003; 98(1):86-93.

15. Gafter-Gvili A, Fraser A, Paul M, Vidal L, Lawrie TA, van de Wetering MD, et al. Antibiotic prophylaxis for bacterial infections in afebrile neutropenic patients following chemotherapy. Cochrane Database Syst Rev. 2012 Jan 18;1:CD004386.

16. Laoprasopwattana $K$, Khwanna $T$, Suwankeeree $P$, Sujjanunt T, Tunyapanit W, ChelaeS. Ciprofloxacin reduces occurrence of fever in children with acute leukemia who develop neutropenia during chemotherapy. Pediatr Infect Dis J. 2013; 32(3):e94-8.

17. Yeh TC, Liu HC, Hou JY, Chen KH, Huang TH, Chang CY, et al. Severe infections in children with acute leukemia undergoing intensive chemotherapy can successfully be prevented by ciprofloxacin, voriconazole, or micafungin prophylaxis. Cancer 2014; 120 (8):1255-62.

18. Inaba $H$, Gaur $A$, Cao X, Flynn $P$, Pounds $S$, Avutu V, et al. Feasibility, efficacy, and adverse effects of outpatient antibacterial prophylaxis in children with acute myeloid leukemia. Cancer 2014; 120(13):1985-92.

19. Samarasinghe $S$, Webb D. How I manage aplastic anaemia in children. British Journal of Haematology 2012; 157(1): 26-40.

20. Williams DA, Bennett $C$, Bertuch A, Bessler $M$, Coates $\mathrm{T}$, Corey $\mathrm{S}$, et al. Diagnosis and treatment of pediatric acquired aplastic anemia (AAA): an initial survey of the North American Pediatric Aplastic Anemia Consortium (NAPAAC). Pediatr Blood Cancer. 2014; 61(5):869-74.

21. Hartung $\mathrm{H}$, Olson $\mathrm{T}$, Bessler M. Acquired Aplastic Anemia in Children. Pediatr Clin North Am. 2013; 60(6): 13111336.
Prevention and management of infections in children with severe aplastic anemia

\section{ABSTRACT}

Aplastic anemia (AA) is a rare condition in children. Hematopoietic stem cell transplantation (HSCT) is the treatment of choice for severe idiopatic AA. Survival in severe AA has markedly improved in the past decades due to advances in HSCT, immunosuppressive and biologic drugs, as well as supportive care. Since bacterial and fungal infections are one of the principal causes of morbidity and mortality in $A A$, one of the main challenges is to prevent the appearance of infections associated with severe and prolonged neutropenia. Most guidelines of treatment and prophylaxis are based on expert recommendations. Given the lack of controlled studies in children with $A A$, most recommendations of empiric treatment rely on guidelines for febrile-neutropenia management in hemato-oncologycal patients. A great variability exists in the use of antimicrobials for primary prophylaxis among different institutions. Due to the fact that patients with severe and prolonged AA present high incidence of filamentous fungal infections, an adecuate antifungal prophylaxis is recommended. In the case of severe lymphopenia, prophylaxis against $\mathrm{P}$ jirovecii should also be considered. Recommendations in prophylaxis and early treatment of infections in severe pediatric $A A$ are detailed.

Keywords: aplastic anemia in children, antimicrobial prophylaxis, prevention of infection, empiric therapy of fever. 